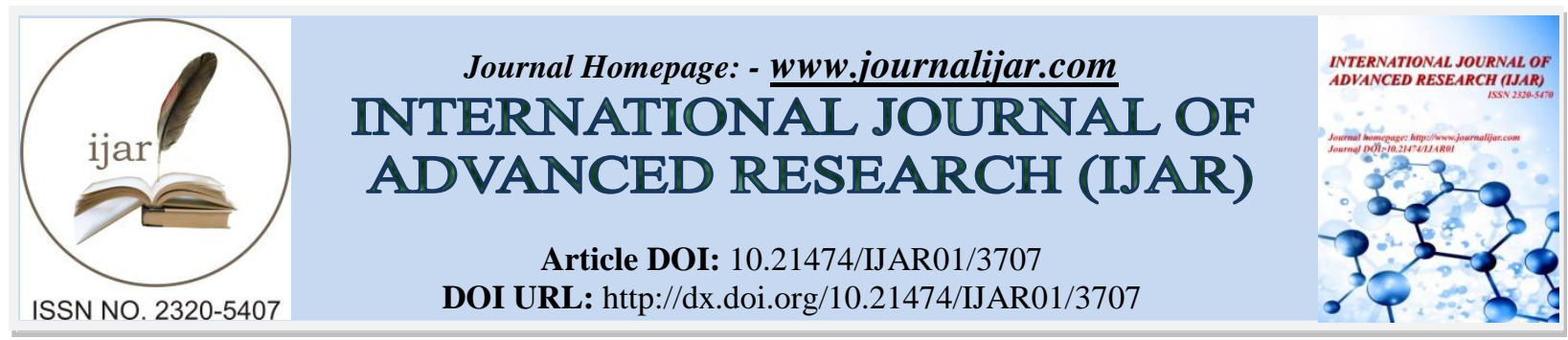

RESEARCH ARTICLE

\title{
DECLINED MORPHOLOGY IN TOBACCO ( Nicotiana tobacum L. ) DUE TO CADMIUM STRESS.
}

*K.Chitra and P.S. Sharavanan ${ }^{1}$.

1. Assistant Professor, Department of Botany, Bharathiar university, Coimbatore-641 046.

2. Associate professor, Department of Botany, Thiru Govindasamy Govt. Arts College, Tindivanam-604 202, Tamilnadu, India

\section{Manuscript Info}

Manuscript History

Received: 15 January 2017

Final Accepted: 02 February 2017

Published: March 2017

Key words:-

Cadmium, tobacco and morphological parameters.

\begin{abstract}
Cadmium is one of the most toxic heavy metal in the environment. Cadmium presence in soil can cause serious problem to all organisms through food chain. The present study was conducted through pot culture experiment to estimate the effect of cadmium $(10,25,50,75$, $100, \& 200 \mathrm{mg} \mathrm{kg}-1$ soil) on the morphological parameters (root length, shoot length, number of leaves, total leaf area, fresh and dry weight of root, stem, leaf) of tobacco. The values were recorded on $30^{\text {th }}, 60^{\text {th }}$, $90^{\text {th }}, 120^{\text {th }}, 150^{\text {th }}$ and $180^{\text {th }}$ days of tobacco. For all the morphological parameters, the highest values were recorded in control. Due to increase in cadmium concentrations all the morphological parameters were decreased from $10 \mathrm{mg} \mathrm{kg}^{-1}$ to $200 \mathrm{mg} \mathrm{kg}^{-1}$ of soil.
\end{abstract}

Copy Right, IJAR, 2017,. All rights reserved.

\section{Introduction:-}

Due to globalization and industrialization, heavy metal (HM) pollution is one of the most important and widespread environmental problem of the modern world, due to its hazardous and toxic effects on all living organisms. Heavy metal pollution is a serious environmental problem because heavy metals are non-biodegradable and persistent in nature $[10,11]$.

Heavy metals are defined as a group of metals and metalloids with atomic density higher than $5 \mathrm{~g} \mathrm{~cm}-3$. The remediation of heavy metal contaminated soil requires the removal of toxic metal from contaminated areas. Heavy metals were important environmental pollutants particularly in areas where there was a high anthropogenic pressure, but they also occur naturally (Sanità and Gabbrielli, 1999). Anthropogenic cadmium contamination often results from mining or smelting of metal ores, but cadmium is also released into the environment by power stations, heating systems, waste incinerators, urban traffic, and cement factories and as a by-product of phosphate fertilizers. Use of sewage sludges as fertilizers has further contributed to a significant contamination of agricultural soils. Cadmium presence in the soil can cause serious problems to all organisms. Although non-essential for plant growth, when bio available, cadmium is readily taken up by roots and translocated into aerial organs where it can accumulate to high levels. The most apparent visible symptoms of cadmium toxicity in plants are retardation of plant growth, chlorosis and stunting (Das et al., 1997). The present investigations was carried out to analyse the changes in growth parameters such as, root and shoot length, number of leaves and total leaf area, fresh and dry weight of root, stem, leaf of tobacco plants due to cadmium toxicity. 


\section{Materials and Methods:- \\ Seed Materials:-}

The seeds of tobacco were procured from Central Tobacco Research Institute, Research Station, Vedasandur, Dindugal Dt, Tamilnadu. The duration of the crop was 180 days. Seeds of uniform size, colour and weight were chosen for the experiments. Cadmium and other chemicals used in this investigation were analytical grade. Cadmium was used in the form of chloride.

\section{Pot Culture Experiments:-}

The experiments were performed during the months of December 2011 to May 2012. Tobacco plants were grown in pots in untreated soil (control) and in soil to which cadmium had been applied (0, 10, 25, 50, 75,100 and $200 \mathrm{mg} \mathrm{kg}^{-1}$ of soil). The inner surface of pots was lined with a polythene sheet. Each pot contained $3.00 \mathrm{~kg}$ of air dried soil. Cadmium as chloride $\left(\mathrm{CdCl}_{2} 21 / 2\right.$ $\mathrm{H}_{2} \mathrm{O}$ ) were finely powdered and applied to the surface soil. This was then thoroughly mixed with the soil and 1 gram of seeds was sown in each pot. All pots were watered to field capacity twice a day. Plants were thinned to a maximum of three per pot after a week of germination. The treatments were replicated five times in a completely randomized block design.

\section{Samplimg:-}

Plant samples were collected at random, at regular intervals $\left(30,60,90,120,150\right.$ and $180^{\text {th }}$ days) and used for morphological growth parameters. Three plants from each replicate of a pot were analysed for its various parameters and the average was calculated. These mean values of the replicates were used for statistical analysis.

\section{Growth:-}

\section{Root and Shoot Length:-}

The root and shoot length and elongation rate are essential for plants exploring for water and mineral nutrients. In the pot culture experiments there was a gradual decrease in the root and shoot length with an increase in cadmium level 10, 25, 50, 75,100 and $200 \mathrm{mg} \mathrm{kg}^{-1}$ in the soil in all the sampling days. The inhibitory action of excess of cadmium in root and shoot length might be due to reduction in cell division, toxic effect of heavy metals on photosynthesis, respiration and protein synthesis. These obviously contributed to the retardation of normal growth Kupper, et al. (1996). The above results were in agreement with the findings of Chen, et al. (2003) in soybean, Rai, et al. (2005) in Phyllanthus amarus and Xu, et al. (2008) in garlic. Hagemeyer et al. (2002.) and Marcnano et al. (2002) also suggested that the morphological and structural effects caused by metal toxicity in plants was due to decrease in root elongation, root tip damage, decrease in root formation, suppression of elongation growth rate of cells, affecting the ultra cellular structure of meristematic cells and inhibition of the size of plant cells and inter cellular spaces.

\section{Number of Leaves and Total Leaf Area:-}

Number of leaves and total leaf area were decreased due to the increase of cadmium concentrations in all the sampling days. Number of leaves, branches and leaf area decreased as the concentration of metal increased. Strong decrease in leaf area was correlated to accumulation of chlorophyll pigments as disturb integration of chlorophyll molecules into stable complex (Skkorzynska Polit and Baszynski, 1997). Similar findings have been reported by Mehindirata et al. (2000)

\section{Fresh and Dry weight:-}

Fresh and dry weight of various parts of the tobacco plants showed a marked decline with the increase in cadmium level in the soil in all the sampling days. Hédiji et al. (2010) reported fresh weight reduction of Solanum lycopersicum under high $\mathrm{Cd}$ levels. There was a progressive fall in the dry matter yield of root, shoot and leaves with the corresponding increase in cadmium (10-200 mg kg-1) level in the soil, in all the sampling days. Similar results were obtained by several authors in a number of plants such as Vitoria et al., (2001) in radish, Kim et al., (2002) in cabbage and lettuce, Zhang et al., (2002) in wheat, Shukla et al., (2003) in wheat, Rai et al., (2005) in Phyllanthus amarus. Abdo, Fatma (2008) in soybean. 
Table 1:- Effect of various concentration of cadmium on root length $\left(\mathrm{cm} \mathrm{plant}^{-1}\right)$ of tobacco (Nicotiana tobacum (L.).

\begin{tabular}{|c|c|c|c|c|c|c|}
\hline $\begin{array}{c}\text { Cadmium } \\
\begin{array}{c}\text { added in the soil } \\
\left(\mathbf{m g ~ k g}^{-1}\right)\end{array}\end{array}$ & $\mathbf{3 0}$ & $\mathbf{6 0}$ & $\mathbf{9 0}$ & $\mathbf{1 2 0}$ & $\mathbf{1 5 0}$ & $\mathbf{1 8 0}$ \\
\cline { 2 - 6 } Control & 3.20 & 49.30 & 65.72 & 76.34 & 82.54 & 85.69 \\
\hline $\mathbf{1 0}$ & 3.00 & 46.32 & 62.35 & 72.58 & 77.25 & 80.65 \\
& $(-6.25)$ & $(-6.04)$ & $(-5.13)$ & $(-4.93)$ & $(-6.41)$ & $(-5.88)$ \\
\hline $\mathbf{2 5}$ & 2.80 & 43.67 & 58.46 & 70.32 & 74.36 & 76.39 \\
& $(-12.50)$ & $(-11.44)$ & $(-11.05)$ & $(-7.89)$ & $(-9.87)$ & $(-10.85)$ \\
\hline $\mathbf{5 0}$ & 2.40 & 35.24 & 52.40 & 67.42 & 72.32 & 75.42 \\
& $(-25.00)$ & $(-28.52)$ & $(-20.27)$ & $(-11.68)$ & $(-12.38)$ & $(-11.99)$ \\
\hline $\mathbf{7 5}$ & 2.10 & 32.45 & 49.72 & 62.35 & 66.54 & 69.24 \\
& $(-34.38)$ & $(-34.18)$ & $(-24.35)$ & $(-18.33)$ & $(-19.38)$ & $(-19.20)$ \\
\hline $\mathbf{1 0 0}$ & 2.00 & 24.65 & 42.65 & 56.49 & 59.22 & 63.25 \\
& $(-37.50)$ & $(-50.00)$ & $(-35.10)$ & $(-23.00)$ & $(-28.25)$ & $(-26.19)$ \\
\hline $\mathbf{2 0 0}$ & 1.80 & 21.44 & 36.75 & 52.75 & 56.33 & 59.35 \\
& $(-43.75)$ & $(-56.51)$ & $(-44.08)$ & $(-30.90)$ & $(-31.75)$ & $(-30.74)$ \\
\hline
\end{tabular}

Table 2:- Effect of different treantments of cadmium on shoot length $\left(\mathrm{cm} \mathrm{plant}^{-1}\right)$ of tobacco (Nicotiana tobacum (L.).

\begin{tabular}{|c|c|c|c|c|c|c|}
\hline \multirow{2}{*}{$\begin{array}{l}\text { Cadmium added } \\
\text { in the soil } \\
\left(\mathrm{mg} \mathrm{kg}^{-1}\right)\end{array}$} & \multicolumn{6}{|c|}{ Sampling days } \\
\hline & 30 & 60 & 90 & 120 & 150 & 180 \\
\hline Control & 5.40 & 28.00 & 39.67 & 54.23 & 58.13 & 62.56 \\
\hline 10 & $\begin{array}{c}5.10 \\
(-5.56)\end{array}$ & $\begin{array}{c}27.78 \\
(-0.79)\end{array}$ & $\begin{array}{c}37.63 \\
(-5.14)\end{array}$ & $\begin{array}{c}50.28 \\
(-7.28)\end{array}$ & $\begin{array}{c}56.73 \\
(-2.41)\end{array}$ & $\begin{array}{c}58.84 \\
(-5.95)\end{array}$ \\
\hline 25 & $\begin{array}{c}4.80 \\
(-11.11)\end{array}$ & $\begin{array}{c}25.18 \\
(-10.07)\end{array}$ & $\begin{array}{c}34.56 \\
(-12.88)\end{array}$ & $\begin{array}{c}46.80 \\
(-13.70)\end{array}$ & $\begin{array}{c}52.19 \\
(-10.22)\end{array}$ & $\begin{array}{c}55.80 \\
(-10.81)\end{array}$ \\
\hline 50 & $\begin{array}{c}4.20 \\
(-22.22) \\
\end{array}$ & $\begin{array}{c}22.65 \\
(-19.11) \\
\end{array}$ & $\begin{array}{c}31.23 \\
(-21.28) \\
\end{array}$ & $\begin{array}{c}43.39 \\
(-19.99) \\
\end{array}$ & $\begin{array}{c}48.49 \\
-19.58)\end{array}$ & $\begin{array}{c}52.50 \\
(-16.08)\end{array}$ \\
\hline 75 & $\begin{array}{c}4.00 \\
(-25.93) \\
\end{array}$ & $\begin{array}{c}17.00 \\
(-39.29)\end{array}$ & $\begin{array}{c}26.54 \\
(-33.10)\end{array}$ & $\begin{array}{c}34.69 \\
(-36.03)\end{array}$ & $\begin{array}{c}38.67 \\
(-33.48) \\
\end{array}$ & $\begin{array}{c}45.39 \\
(-27.45) \\
\end{array}$ \\
\hline 100 & $\begin{array}{c}2.60 \\
(-51.85)\end{array}$ & $\begin{array}{c}13.32 \\
(-52.43)\end{array}$ & $\begin{array}{c}23.45 \\
(-40.89)\end{array}$ & $\begin{array}{c}31.36 \\
(-42.17)\end{array}$ & $\begin{array}{c}36.52 \\
(-37.18)\end{array}$ & $\begin{array}{c}39.24 \\
(-37.28)\end{array}$ \\
\hline 200 & $\begin{array}{c}2.80 \\
(-48.15)\end{array}$ & $\begin{array}{c}11.34 \\
(-59.50)\end{array}$ & $\begin{array}{c}19.32 \\
(-51.30)\end{array}$ & $\begin{array}{c}28.65 \\
(-47.17)\end{array}$ & $\begin{array}{c}33.56 \\
(-42.27)\end{array}$ & $\begin{array}{c}34.69 \\
(-44.55)\end{array}$ \\
\hline
\end{tabular}

Table 3:- Effect of various cadmium concentration of leaf number $\left(\right.$ plant $\left.^{-1}\right)$ of tobacco (Nicotiana tobacum .(L.)

\begin{tabular}{|c|c|c|c|c|c|c|}
\hline $\begin{array}{c}\text { Cadmium added in } \\
\text { the soil }\left(\mathbf{m g ~ k g}^{-1}\right)\end{array}$ & $\mathbf{3 0}$ & $\mathbf{6 0}$ & $\mathbf{9 0}$ & $\mathbf{1 2 0}$ & $\mathbf{1 5 0}$ & $\mathbf{1 8 0}$ \\
\cline { 2 - 7 } Control & 5.00 & 9.32 & 13.58 & 19.34 & 18.44 & 17.45 \\
\hline $\mathbf{1 0}$ & 4.00 & 8.65 & 12.62 & 18.66 & 17.24 & 16.58 \\
& $(-20.00)$ & $(-7.19)$ & $(-7.07)$ & $(-3.52)$ & $(-6.51)$ & $(-4.99)$ \\
\hline $\mathbf{2 5}$ & 4.00 & 8.00 & 12.24 & 18.00 & 16.63 & 16.30 \\
& $(-20.00)$ & $(-14.19)$ & $(-9.87)$ & $(-6.93)$ & $(-9.82)$ & $(-6.59)$ \\
\hline $\mathbf{5 0}$ & 3.00 & 7.34 & 11.24 & 16.45 & 15.84 & 15.08 \\
& $(-40.00)$ & $(-21.24)$ & $(-17.23)$ & $(-14.94)$ & $(-14.10)$ & $(-13.58)$ \\
\hline $\mathbf{7 5}$ & 2.00 & 7.00 & 9.52 & 15.76 & 15.22 & 13.34 \\
& $(-60.00)$ & $(-34.89)$ & $(-29.90)$ & $(-18.51)$ & $(-17.46)$ & $(-23.55)$ \\
\hline $\mathbf{1 0 0}$ & 2.00 & 6.33 & 8.80 & 15.49 & 13.39 & 12.66 \\
& $(-60.00)$ & $(-32.08)$ & $(-35.20)$ & $(-19.91)$ & $(-27.39)$ & $(-27.45)$ \\
\hline $\mathbf{2 0 0}$ & 2.00 & 5.82 & 8.24 & 13.62 & 12.54 & 12.09 \\
& $(-60.00)$ & $(-37.55)$ & $(-39.32)$ & $(-29.58)$ & $(-32.00)$ & $(-30.72)$ \\
\hline
\end{tabular}


Table 4:- Effect of cadmium on total leaf area $\left(\mathrm{cm}^{2}\right)$ of tobacco (Nicotiana tobacum (L.).

\begin{tabular}{|c|c|c|c|c|c|c|}
\hline \multirow{2}{*}{$\begin{array}{l}\text { Cadmium added in the soil } \\
\left(\mathrm{mg} \mathrm{kg}^{-1}\right)\end{array}$} & \multicolumn{6}{|c|}{ Sampling days } \\
\hline & 30 & 60 & 90 & 120 & 150 & 180 \\
\hline Control & 62.14 & 657.49 & 936.05 & 1056.45 & 865.32 & 788.90 \\
\hline 10 & $\begin{array}{c}60.01 \\
(-3.43)\end{array}$ & $\begin{array}{l}624.52 \\
(-5.01)\end{array}$ & $\begin{array}{l}924.69 \\
(-1.21)\end{array}$ & $\begin{array}{l}988.40 \\
(-6.44)\end{array}$ & $\begin{array}{l}846.56 \\
(-2.17)\end{array}$ & $\begin{array}{l}727.13 \\
(-7.83)\end{array}$ \\
\hline 25 & $\begin{array}{c}57.57 \\
(-7.35)\end{array}$ & $\begin{array}{l}595.43 \\
(-9.44)\end{array}$ & $\begin{array}{l}861.13 \\
(-8.00)\end{array}$ & $\begin{array}{l}934.25 \\
(-11.57)\end{array}$ & $\begin{array}{l}812.30 \\
(-6.13)\end{array}$ & $\begin{array}{l}677.29 \\
(-14.15)\end{array}$ \\
\hline 50 & $\begin{array}{c}57.38 \\
(-7.66)\end{array}$ & $\begin{array}{c}546.80 \\
(-16.84)\end{array}$ & $\begin{array}{c}836.72 \\
(-10.61)\end{array}$ & $\begin{array}{c}908.57 \\
(-14.00)\end{array}$ & $\begin{array}{c}768.27 \\
(-11.22)\end{array}$ & $\begin{array}{c}643.15 \\
(-18.48)\end{array}$ \\
\hline 75 & $\begin{array}{c}43.82 \\
(-29.48)\end{array}$ & $\begin{array}{c}510.87 \\
(-22.30)\end{array}$ & $\begin{array}{l}741.98 \\
(-20.73)\end{array}$ & $\begin{array}{l}897.23 \\
(-15.07)\end{array}$ & $\begin{array}{l}754.35 \\
(-12.82)\end{array}$ & $\begin{array}{c}609.24 \\
(-22.77)\end{array}$ \\
\hline 100 & $\begin{array}{c}37.11 \\
(-40.28)\end{array}$ & $\begin{array}{l}489.64 \\
(-25.53)\end{array}$ & $\begin{array}{l}683.76 \\
(-26.95)\end{array}$ & $\begin{array}{c}765.27 \\
(-27.56)\end{array}$ & $\begin{array}{l}723.40 \\
(-16.40)\end{array}$ & $\begin{array}{c}570.12 \\
(-27.73)\end{array}$ \\
\hline 200 & $\begin{array}{c}28.72 \\
(-53.78)\end{array}$ & $\begin{array}{l}453.16 \\
(-31.08)\end{array}$ & $\begin{array}{r}548.97 \\
(-41.35)\end{array}$ & $\begin{array}{c}738.67 \\
(-30.08)\end{array}$ & $\begin{array}{l}698.24 \\
(-19.31)\end{array}$ & $\begin{array}{c}534.25 \\
(-32.28)\end{array}$ \\
\hline
\end{tabular}

Table 5:- Effect of cadmium on root fresh weight (g plant ${ }^{-1}$ ) of tobacco (Nicotiana tobacum (L.).

\begin{tabular}{|c|c|c|c|c|c|c|}
\hline \multirow{2}{*}{$\begin{array}{c}\text { Cadmium adde in } \\
\text { the soil (mg kg }\end{array}$} & \multicolumn{7}{|c|}{ Sampling days } \\
\cline { 2 - 7 } & $\mathbf{3 0}$ & $\mathbf{6 0}$ & $\mathbf{9 0}$ & $\mathbf{1 2 0}$ & $\mathbf{1 5 0}$ & $\mathbf{1 8 0}$ \\
\hline Control & 3.380 & 5.628 & 8.212 & 10.504 & 12.720 & 14.792 \\
\hline \multirow{2}{*}{$\mathbf{1 0}$} & 3.093 & 5.231 & 8.120 & 10.345 & 12.546 & 14.025 \\
& $(-8.49)$ & $(-7.05)$ & $(-1.12)$ & $(-1.51)$ & $(-1.37)$ & $(-5.19)$ \\
\hline \multirow{2}{*}{$\mathbf{2 5}$} & 2.856 & 4.674 & 7.459 & 10.256 & 12.190 & 13.564 \\
& $(-15.50)$ & $(-16.95)$ & $(-9.17)$ & $(-2.36)$ & $(-4.17)$ & $(-8.30)$ \\
\hline \multirow{2}{*}{$\mathbf{5 0}$} & 2.530 & 4.267 & 7.235 & 10.178 & 11.589 & 12.785 \\
& $(-25.15)$ & $(-24.18)$ & $(-11.90)$ & $(-3.10)$ & $(-8.89)$ & $(-13.57)$ \\
\hline \multirow{2}{*}{$\mathbf{7 5}$} & 2.324 & 4.067 & 7.189 & 09.467 & 11.280 & 11.568 \\
& $(-31.24)$ & $(-27.74)$ & $(-12.46)$ & $(-9.87)$ & $(-11.32)$ & $(-21.80)$ \\
\hline \multirow{2}{*}{$\mathbf{1 0 0}$} & $2 . .102$ & 3.780 & 6.984 & 8.432 & 10.453 & 10.482 \\
& $(-37.81)$ & $(-32.84)$ & $(-14.95)$ & $(-19.73)$ & $(-17.82)$ & $(-29.17)$ \\
\hline \multirow{2}{*}{$\mathbf{2 0 0}$} & 1.345 & 3.455 & 6.745 & 8.345 & 10.260 & 9.680 \\
& $(-60.21)$ & $(-38.61)$ & $(-17.86)$ & $(-20.55)$ & $(-19.34)$ & $(-34.56)$ \\
\hline
\end{tabular}

Table 6:- Impact of cadmium on stem fresh weight $\left(\mathrm{g} \mathrm{plant}^{-1}\right)$ of tobacco (Nicotiana tobacum (L.).

\begin{tabular}{|c|c|c|c|c|c|c|}
\hline $\begin{array}{c}\text { Cadmium added in } \\
\text { the soil }\left(\mathbf{m g ~ k g}^{-1}\right)\end{array}$ & \multicolumn{6}{|c|}{ Sampling days } \\
\cline { 2 - 7 } & $\mathbf{3 0}$ & $\mathbf{6 0}$ & $\mathbf{9 0}$ & $\mathbf{1 2 0}$ & $\mathbf{1 5 0}$ & $\mathbf{1 8 0}$ \\
\hline Control & 5.435 & 17.028 & 35.635 & 66.849 & 73.760 & 80.534 \\
\hline $\mathbf{1 0}$ & 5.178 & 16.324 & 35.097 & 63.765 & 71.005 & 78.546 \\
& $(-4.73)$ & $(-4.13)$ & $(-1.51)$ & $(-4.61)$ & $(-3.74)$ & $(-2.47)$ \\
\hline $\mathbf{2 5}$ & 4.325 & 14.455 & 32.678 & 60.324 & 69.425 & 74.750 \\
& $(-20.42)$ & $(-15.11)$ & $(-8.30)$ & $(-9.76)$ & $(-5.88)$ & $(-7.18)$ \\
\hline $\mathbf{5 0}$ & 3.754 & 13.769 & 30.537 & 57.920 & 66.439 & 72.435 \\
& $(-30.93)$ & $(-19.14)$ & $(-14.31)$ & $(-13.36)$ & $(-9.93)$ & $(-10.06)$ \\
\hline $\mathbf{7 5}$ & 3.432 & 13.769 & 28.980 & 55.345 & 63.761 & 70.523 \\
& $(-36.85)$ & $(-19.14)$ & $(-18.68)$ & $(-17.21)$ & $(-13.56)$ & $(-12.43)$ \\
\hline $\mathbf{1 0 0}$ & 3.098 & 11.560 & 25.008 & 52.894 & 62.835 & 69.425 \\
& $(-43.00)$ & $(-32.11)$ & $(-29.82)$ & $(-20.88)$ & $(-14.81)$ & $(-13.79)$ \\
\hline $\mathbf{2 0 0}$ & 2.270 & 10.426 & 23.349 & 49.670 & 60.033 & 67.213 \\
& $(-58.23)$ & $(-38.77)$ & $(-34.48)$ & $(-25.70)$ & $(-18.61)$ & $(-16.54)$ \\
\hline
\end{tabular}


Table 7:- Influence of different treatments of cadmium on leaf fresh weight $\left(\mathrm{g} \mathrm{plant}^{-1}\right.$ ) of tobacco (Nicotiana tobacum (L.).

\begin{tabular}{|c|c|c|c|c|c|c|}
\hline \multirow{2}{*}{$\begin{array}{c}\text { Cadmium added in } \\
\text { the soil }\left(\mathbf{m g ~ k g}^{-1}\right)\end{array}$} & \multicolumn{7}{|c|}{ Sampling days } \\
\cline { 2 - 7 } & $\mathbf{3 0}$ & $\mathbf{6 0}$ & $\mathbf{9 0}$ & $\mathbf{1 2 0}$ & $\mathbf{1 5 0}$ & $\mathbf{1 8 0}$ \\
\hline Control & 7.692 & 19.296 & 34.938 & 53.342 & 44.890 & 40.453 \\
\hline $\mathbf{1 0}$ & 7.420 & 17.650 & 32.540 & 49.430 & 40.563 & 38.546 \\
& $(-3.54)$ & $(-8.53)$ & $(-6.86)$ & $(-7.33)$ & $(-9.64)$ & $(-4.71)$ \\
\hline $\mathbf{2 5}$ & 6.765 & 16.548 & 30.123 & 45.367 & 38.425 & 35.638 \\
& $(-12.05)$ & $(-14.24)$ & $(-13.78)$ & $(-14.95)$ & $(-14.40)$ & $(-11.90)$ \\
\hline $\mathbf{5 0}$ & 6.324 & 14.670 & 28.324 & 44.215 & 36.430 & 32.879 \\
& $(-17.78)$ & $(-23.97)$ & $(-18.93)$ & $(-17.11)$ & $(18.85)$ & $(-18.72)$ \\
\hline $\mathbf{7 5}$ & 5.798 & 13.567 & 26.365 & 40.652 & 34.630 & 30.098 \\
& $(-24.62)$ & $(-29.69)$ & $(-24.54)$ & $(-23.79)$ & $(-22.86)$ & $(-25.60)$ \\
\hline $\mathbf{1 0 0}$ & 4.430 & 12.646 & 24.310 & 37.638 & 30.768 & 27.432 \\
& $(-42.41)$ & $(-34.46)$ & $(-30.44)$ & $(-29.44)$ & $(-31.46)$ & $(-32.19)$ \\
\hline $\mathbf{2 0 0}$ & 3.544 & 10.780 & 22.645 & 35.319 & 28.429 & 25.560 \\
& $(-53.93)$ & $(-44.13)$ & $(-35.19)$ & $(-33.79)$ & $(-36.67)$ & $(-36.82)$ \\
\hline
\end{tabular}

Table 8:- Various treatment of cadmium on root dry weight $\left(\mathrm{g} \mathrm{plant}^{-1}\right)$ of tobacco (Nicotiana tobacum (L.) .

\begin{tabular}{|c|c|c|c|c|c|c|}
\hline \multirow{2}{*}{$\begin{array}{c}\text { Cadmium added in } \\
\text { the soil (mg kg-1 }\end{array}$} & \multicolumn{7}{|c|}{ Sampling days } \\
\cline { 2 - 7 } Control & $\mathbf{3 0}$ & $\mathbf{6 0}$ & $\mathbf{9 0}$ & $\mathbf{1 2 0}$ & $\mathbf{1 5 0}$ & $\mathbf{1 8 0}$ \\
\hline $\mathbf{1 0}$ & 0.845 & 1.657 & 2.053 & 2.876 & 3.180 & 3.698 \\
& 0.765 & 1.465 & 1.596 & 2.563 & 2.897 & 3.182 \\
& $(-9.47)$ & $(-11.59)$ & $(-22.26)$ & $(-10.88)$ & $(-8.90)$ & $(-13.95)$ \\
\hline $\mathbf{2 5}$ & 0.709 & 1.089 & 1.256 & 2.239 & 2.569 & 2.845 \\
& $(-16.09)$ & $(-34.28)$ & $(-38.82)$ & $(-22.15)$ & $(-19.21)$ & $(-23.07)$ \\
\hline $\mathbf{5 0}$ & 0.675 & 0.960 & 1.189 & 1.980 & 2.165 & 2.654 \\
& $(-20.12)$ & $(-42.06)$ & $(-42.08)$ & $(-31.15)$ & $(-31.92)$ & $(-28.23)$ \\
\hline $\mathbf{7 5}$ & 0.496 & 0.827 & 1.025 & 1.635 & 1.988 & 2.267 \\
& $(-41.30)$ & $(-50.09)$ & $(-50.07)$ & $(-43.15)$ & $(-37.48)$ & $(-38.70)$ \\
\hline $\mathbf{1 0 0}$ & 0.463 & 0.724 & 0.898 & 1.298 & 1.760 & 1.918 \\
& $(-45.21)$ & $(-56.31)$ & $(-56.26)$ & $(-54.87)$ & $(-44.65)$ & $(-48.13)$ \\
\hline $\mathbf{2 0 0}$ & 0.375 & 0.549 & 0.617 & 1.056 & 1.562 & 1.875 \\
& $(-55.62)$ & $(-66.87)$ & $(-69.95)$ & $(-63.28)$ & $(-50.88)$ & $(-49.30)$ \\
\hline
\end{tabular}

Table 9:- Different treatment of cadmium on stem dry weight $\left(\mathrm{g} \mathrm{plant}^{-1}\right)$ of tobacco (Nicotiana tobacum (L.).

\begin{tabular}{|c|c|c|c|c|c|c|}
\hline $\begin{array}{c}\text { Cadmium added in } \\
\text { the soil (mg kg }\end{array}$ & \multicolumn{6}{|c|}{ Sampling days } \\
\cline { 2 - 7 } Control & $\mathbf{3 0}$ & $\mathbf{6 0}$ & $\mathbf{9 0}$ & $\mathbf{1 2 0}$ & $\mathbf{1 5 0}$ & $\mathbf{1 8 0}$ \\
\hline $\mathbf{1 0}$ & 2.860 & 7.684 & 12.456 & 15.675 & 18.467 & 21.019 \\
\hline $\mathbf{2 5}$ & 2.389 & 7.398 & 12.180 & 15.270 & 18.167 & 20.867 \\
& $(-16.47)$ & $(-3.72)$ & $(-2.22)$ & $(-2.58)$ & $(-1.62)$ & $(-0.72)$ \\
\hline $\mathbf{5 0}$ & 2.160 & 7.212 & 11.678 & 15.055 & 17.985 & 19.539 \\
& $(-24.48)$ & $(-6.14)$ & $(-6.25)$ & $(-3.96)$ & $(-2.61)$ & $(-7.04)$ \\
\hline $\mathbf{7 5}$ & 1.989 & 6.389 & 11.398 & 14.784 & 17.580 & 18.480 \\
& $(-30.45)$ & $(-16.85)$ & $(-8.49)$ & $(-5.68)$ & $(-4.80)$ & $(-12.08)$ \\
\hline $\mathbf{1 0 0}$ & 1.670 & 6.318 & 11.267 & 14.470 & 17.360 & 18.384 \\
& $(-41.61)$ & $(-17.78)$ & $(-9.55)$ & $(-7.69)$ & $(-5.99)$ & $(-12.54)$ \\
\hline $\mathbf{2 0 0}$ & 1.467 & 6.265 & 11.098 & 14.354 & 16.936 & 17.934 \\
& $(-48.71)$ & $(-18.47)$ & $(-10.90)$ & $(-8.43)$ & $(-8.29)$ & $(-14.68)$ \\
\hline & 1.082 & 5.545 & 10.865 & 13.088 & 16.680 & 17.685 \\
& $(-62.17)$ & $(-27.84)$ & $(-12.77)$ & $(-16.50)$ & $(-9.68)$ & $(-15.86)$ \\
\hline
\end{tabular}


Table 10:- Efficacy of cadmium treatment on leaf dry weight (g plant ${ }^{-1}$ ) of tobacco (Nicotiana tobacum (L.).

\begin{tabular}{|c|c|c|c|c|c|c|}
\hline $\begin{array}{c}\text { Cadmium added in } \\
\text { the soil (mg kg-1 }\end{array}$ & \multicolumn{6}{|c|}{ Sampling days } \\
\cline { 2 - 7 } Control & $\mathbf{3 0}$ & $\mathbf{6 0}$ & $\mathbf{9 0}$ & $\mathbf{1 2 0}$ & $\mathbf{1 5 0}$ & $\mathbf{1 8 0}$ \\
\hline $\mathbf{1 0}$ & 2.564 & 6.435 & 11.546 & 17.756 & 15.762 & 13.453 \\
& 2.380 & 6.319 & 11.345 & 17.534 & 15.564 & 13.235 \\
& $(-7.18)$ & $(-1.80)$ & $(-1.74)$ & $(-1.25)$ & $(-1.26)$ & $(-1.62)$ \\
\hline $\mathbf{2 5}$ & 2.245 & 6.212 & 10.689 & 17.231 & 14.432 & 13.154 \\
& $(-12.44)$ & $(-3.47)$ & $(-7.42)$ & $(-2.96)$ & $(-8.44)$ & $(-2.22)$ \\
\hline $\mathbf{5 0}$ & 1.987 & 5.289 & 10.453 & 16.564 & 14.245 & 13.058 \\
& $(-22.50)$ & $(-17.81)$ & $(-9.47)$ & $(-6.71)$ & $(-9.62)$ & $(-2.94)$ \\
\hline $\mathbf{7 5}$ & 1.589 & 5.167 & 10.156 & 15.780 & 14.180 & 11.890 \\
& $(-38.03)$ & $(-19.70)$ & $(-12.04)$ & $(-11.13)$ & $(-10.04)$ & $(-11.62)$ \\
\hline $\mathbf{1 0 0}$ & 1.420 & 5.098 & 10.098 & 15.324 & 13.657 & 11.134 \\
& $(-44.62)$ & $(-20.78)$ & $(-12.54)$ & $(-13.70)$ & $(-13.35)$ & $(-17.24)$ \\
\hline $\mathbf{2 0 0}$ & 0.982 & 4.545 & 09.437 & 15.160 & 13.423 & 10.985 \\
& $(-61.70)$ & $(-29.37)$ & $(-18.27)$ & $(-14.62)$ & $(-14.84)$ & $(-18.35)$ \\
\hline
\end{tabular}

\section{Conclusion:-}

In the present study tobacco plants were grown in control and different levels of cadmium were assessed. Results showed that all the morphological parameters of tobacco were decreased as cadmium level increased. The results indicated that all the morphological parameters dependent on both the different levels of cadmium accumulation and biomass of the plants, when the tobacco plants were grown in cadmium contaminated soil.

\section{References:-}

1. Abdo, Fatma A., 2008. Effect of phosphate dissolving bacteria inoculation and phosphorus fertilization on growth, yield and seed quality of soybean. Egypt. J. Agric. Res., 86(3): 985-1009.

2. Das, P., S. Samantaray, and G.R. Rout. 1997. Studies on Cadmium toxicity in plants. A review. Environ. Poll. 18 (1): 29-36.

3. Hagemeyer, J. and S.W. Breckle. 2002. Trace element stress in roots. In: Y. Waisel, A. Eshel and U. Kafkafi (eds.), Plant roots: the hidden half, 3rd edn. Dekker, New York, pp. 763-785.

4. Hédiji H, Djebali W, Cabasson C, Maucourt M, Baldet P, Pertrand A, Zoghlami LB, Deborde C, Moing A, Brouquisse R, Chaibi W, Gallusci P. Effects of long-term cadmium exposure on growth and metabolomic profile of tomato plants. Ecotoxicol Environ Saf. 2010;73:1965-1974. doi: 10.1016/j.ecoenv.2010.08.014.

5. Khan, S., 2010. Soil and vegetables enrichment with heavy metals from geological sources in Gilgit, northern Pakistan. Ecotoxicology and Environmental Safety, 73: 1820-1827.

6. Kim, W., G.B. Jung, J.S. Lee, J.H. Kim, S.G. Yun and R.D. Park, 2002. Effect of cadmium and arsenic in soils on growth and availability to vegetables. Thailand, $17^{\text {th }}$ WCSS, 14-21.

7. Kupper, H., F. Kupper, and M. Spiller. 1996. Environmental relevance of heavy metal-substituted chlorophylls using the example of water plants. J. Exp. Bot. 47: 259-266.

8. Marcnano, L., I. Carruyo, A. Del Campo, and X. Monteil. 2002. Effect of cadmium on the nucleoli of meristemetic cells of onion Allium cepa L.: An ultra structural study. Environ. Res. 88: 30-35.

9. Mehindirata, S., T.O. Mahmooduzzafar and M. Iqbal: Cadmium induced changes in growth and structure of root and stem of Solanum melongena L. Phytomorphol., 50, 243-251 (2000).

10. Muhammad, S., M.T. Shah and S. Khan, 2011. Health Risk assessment of heavy metals and their source apportionment in drinking water of Kohistan region, northern Pakistan. Microchemical Journal, 98: 334-343.

11. Radwan, M.A. and A.K. 2006. Salama, Market basket survey for some heavy metals in Egyptian fruits and vegetables. Food and Chemical Toxicology, 44: 1273-1278.

12. Rai, V., S. Khatoon, S.S. Bisht, and S. Mehrotra. 2005. Effect of cadmium on growth, ultra morphology of leaf and secondary metabolites of Phyllanthus amarus Schum. and Thonn. Chemosphere. 61:1644-1650.

13. Sanita di Toppi, L. and R. Gabbrielli. 1999. Response to cadmium in higher plants- A review. Env. Exp. Bot. 41: 105-130.

14. Shukla, V.C., J. Singh, P.C. Joshi and P. Kakkar, 2003. Effect of bioaccumulation of cadmium on biomass productivity, essential trace elements, chlorophyll biosynthesis and macromolecules of wheat seedlings. Biol. Trace Element Res., 92: 257-273. 
15. Skorzynska-Polit, E. and T. Baszynski: Differences in sensitivity of photosynthetic apparatus in Cd-stressed bean plants in relation to their age. Pl. Sci., 128, 11-21 (1997).

16. Vitoria A.P., P.J. Pea and R.A. Azevedo, 2001. Antioxidant enzymes responses to cadmium in radish tissues. Phytochem., 57: 701-710.

17. Xu, P., J. Zou, Q. Meng, J. Zou, W. Jiang, and D. Liu. 2008. Effects of Cd2+ on seedling growth of garlic (Allium sativum L.) and selected physiological and biochemical characters. Bioresource Tech. 99 (14): 63726378.

18. Zhang, G., M. Fukami and H. Sekimoto, 2002. Influence of cadmium on mineral concentrations and components in wheat genotypes differing in Cd tolerance at seedling stage. Field Crop Research, 77: 93-98. 